\title{
Preventative treatment with Fluorothiazinon suppressed Acinetobacter baumannii-associated septicemia in mice
}

\author{
Nataliya E. Bondareva $\mathbb{D}^{1}$ - Anna V. Soloveva ${ }^{1} \cdot$ Anna B. Sheremet $\mathbb{D}^{1} \cdot$ Ekaterina A. Koroleva ${ }^{1} \cdot$ Lidiya N. Kapotina ${ }^{1} \cdot$ \\ Elena Y. Morgunova ${ }^{1} \cdot$ Sergei I. Luyksaar $\mathbb{1}^{1} \cdot$ Egor S. Zayakin $^{1} \cdot$ Nailya A. Zigangirova $^{1}$
}

Received: 16 August 2021 / Revised: 7 October 2021 / Accepted: 10 November 2021 / Published online: 21 January 2022

(c) The Author(s), under exclusive licence to the Japan Antibiotics Research Association 2022

\begin{abstract}
The high prevalence of multidrug-resistant Acinetobacter baumannii has emerged as a serious problem in the treatment of nosocomial infections in the past three decades. Recently, we developed a new small-molecule inhibitor belonging to a class of 2,4-disubstituted-4H-[1,3,4]-thiadiazine-5-ones, Fluorothiazinon (FT, previously called CL-55). FT effectively suppressed the T3SS of Chlamydia spp., Pseudomonas aeruginosa, and Salmonella sp. without affecting bacterial growth in vitro. In this study, we describe that prophylactic use of FT for 4 days prior to challenge with resistant clinical isolates of A. baumannii (ABT-897-17 and 52TS19) suppressed septic infection in mice, resulting in improved survival, limited bacteraemia and decreased bacterial load in the organs of the mice. We show that FT had an inhibitory effect on $A$. baumannii biofilm formation in vitro and, to a greater extent, on biofilm maturation. In addition, FT inhibited Acinetobacter isolate-induced death of HeLa cells, which morphologically manifested as apoptosis. The mechanism of FT action on $A$. baumannii is currently being studied. FT may be a promising candidate for the development of a broad-spectrum antivirulence drug to use in the prevention of nosocomial infections.
\end{abstract}

\section{Introduction}

Acinetobacter baumannii is an important pathogen associated with hospital-acquired infections worldwide, with clinical manifestations in the form of nosocomial pneumonia and bacteraemia. The highest density of infections occurs in intensive care units (ICUs) among patients on mechanical ventilation. Due to the worldwide coronavirus disease 2020 (COVID-19) pandemic, many patients are experiencing severe pulmonary symptoms, and the use of mechanical ventilation has increased dramatically. Despite saving lives, the use of mechanical ventilation can lead to ventilator-associated pneumonia with high mortality,

Supplementary information The online version contains supplementary material available at https://doi.org/10.1038/s41429022-00504-y.

Nataliya E. Bondareva

nataliia.d@mail.ru

1 Gamaleya National Research Center of Epidemiology and Microbiology, Ministry of Health Russian Federation, Medical Microbiology, Moscow, Russian Federation especially when the patient is infected with multidrugresistant bacteria [1]. The presence of an endotracheal tube creates ideal conditions for the transmission of Acinetobacter, which effectively adheres to plastic and forms biofilms on its surface.

The most important determinant in the clinical outcome of Acinetobacter infections is antibiotic resistance [2, 3]. It is urgent to find new antimicrobial agents that operate under different principles than traditional antibiotics. In this respect anti-virulence therapy is a promising alternative since instead of killing pathogens, it aims to control pathogen virulence without selective pressure.

In a previous work, we demonstrated that a new smallmolecule inhibitor of the T3SS designated Fluorothiazinon (FT, previously called CL-55), a 2,4-disubstituted-4H$[1,3,4]$-thiadiazine-5-one, effectively suppressed the T3SS of Chlamydia spp., Pseudomonas aeruginosa, and Salmonella sp. in vitro and in vivo but did not affect bacterial growth in vitro. FT effectively controlled $C$. trachomatis serovar D vaginal shedding, ascending infection, and inflammation in the upper genital organs in DBA/2 mice $[4,5]$. In an airway $P$. aeruginosa infection murine model, FT reduced mortality and bacterial loads and decreased lung pathology and systemic inflammation when given as a 
combined prophylaxis-treatment regimen or administered as a therapy started after the onset of infection [6]. In a murine model of acute Salmonella infection, the developed T3SS inhibitor significantly formulated the numbers of Salmonella in the spleen and peritoneal lavages and increased the survival rates in susceptible (BALB/c, I/St) and resistant (A/ $\mathrm{Sn})$ mice. FT suppressed oral Salmonella infection in A/Sn mice when used both therapeutically or prophylactically and led to a significant decrease in bacterial burden in mice [7, 8]. Currently, a formulated drug product with FT as an active ingredient, named Ftortiazinon, is undergoing research in phase 2 clinical trials in Russia (Safety and Efficacy Study of Ftortiazinon in the Treatment of Patients With Complicated Urinary Tract Infections Caused by $P$. aeruginosa, NCT03638830).

Further study of the range of action of FT in preclinical studies showed that the inhibitor suppressed A. baumannii infection in mice. In this study, we describe that prophylactic use of FT for 4 days prior to challenge with resistant clinical isolates of A. baumannii (ABT-897-17 and 52TS19) suppressed septic infection in mice, resulting in improved survival and limited bacteraemia. The level of the key proinflammatory cytokine IL-6 in peritoneal lavage decreased in the FT-treated group at all time points of observation, but in the serum of the FT-treated mice, the IL6 level was significantly decreased $2 \mathrm{~h}$ after challenge. We show that FT had a time-dependent inhibitory effect on $A$. baumannii biofilms and, to a greater extent, on the final formation of the bacterial community and biofilm maturation. In addition, FT inhibited Acinetobacter-induced death of HeLa cells morphologically manifesting as apoptosis. The mechanism of FT action on A. baumannii is currently being studied.

\section{Materials and methods}

\section{Fluorothiazinon}

FT is $N$-(2,4-difluorophenyl)-4(3-ethoxy-4-hydroxybenzyl)5-oxo-5,6-dihydro-4H-[1,3,4]-thiadiazine-2-carboxamide, previously reported as CL-55, and synthesized as previously described [5].

\section{Bacterial strains and growth conditions}

A clinical isolate of A. baumannii, 52TS19, was obtained from the sputum of a patient with pneumonia, and the clinical isolate A. baumannii ABT-897-17 was obtained from the blood of a patient with postoperative abdominal sepsis. The isolates were kindly provided by Dr. Voronina O.L. and Dr. Zhukhovitsky V.G. (NRCEM). The identities of the isolates were additionally confirmed by MALDI-TOF mass spectrometry. The antibiotic sensitivity of isolates was investigated by the disk diffusion method. The isolates were cultured in Luria-Bertani (LB) broth at $37^{\circ} \mathrm{C}$ and stored indefinitely when maintained frozen at $-70^{\circ} \mathrm{C}$ or below.

\section{Mice and ethics statement}

Specific-pathogen-free 8-to-9-week-old female and male $\mathrm{BALB} / \mathrm{c}$ and DBA/2 mice were bred and maintained under conventional conditions at the Animal Facilities of the Gamaleya National Research Center for Epidemiology and Microbiology, Moscow, Russia. The animals were maintained and used in accordance with the recommendations of the National Guidelines, and experimental procedures were approved by the Gamaleya National Research Center Animal Care Committee.

\section{Acinetobacter septic infection model and preventive treatment}

For intraperitoneal inoculation, bacterial strains were grown in LB broth overnight, centrifuged at $6000 \mathrm{rpm}$ for $10 \mathrm{~min}$, resuspended in sterile physiological saline and diluted to the necessary concentration. Mice were injected with $0.5 \mathrm{ml}$ of A. baumannii isolate suspension intraperitoneally using a $1.0 \mathrm{ml}$ syringe with a $30 \mathrm{~g}$ needle at the doses indicated in the Results section. Estimation of the condition of the animals was performed every hour post-infection (p.i.).

The development of the infectious process is characterized by a change in the state of animals, such as weight loss, ruffled fur, poor appetite, loss of coordination and unusual movement, huddling behavior, and low body temperature. Mice that displayed huddling behavior, had a low body temperature and showed poor mobility were weighed every $4 \mathrm{~h}$. When the weight loss exceeded $15 \%$, the mice were euthanized.

FT was administered per os by gavage needle at a dose of $250 \mathrm{mg} \mathrm{kg}^{-1}$ in $0.2 \mathrm{ml} 1 \%$ starch solution once per day for 4 days prior to infection; the comparison groups received $1 \%$ starch solution once per day in the same volume.

\section{Sample collection and processing}

Post-mortem blood samples are taken from the heart ventricle, which was accessed through the upper sternum. Blood samples were collected for quantitative bacteriology and cytokine assays. In addition, to obtain samples for the cytokine assay, the peritoneal cavity was lavaged with $5 \mathrm{ml}$ of sterile saline.

The lung and spleen were removed under aseptic conditions, homogenized in $1 \mathrm{ml}$ of saline on a mechanical homogenizer, and centrifuged for $10 \mathrm{~min}$ at $800 \mathrm{rpm}$ for use in quantitative bacteriology. 
To assess the bacterial infection load, $100 \mu \mathrm{l}$ aliquots of 10 -fold serial dilutions of whole blood and tissue homogenates were cultured on LB agar plates, and the number of viable $A$. baumannii in the corresponding samples was quantified. The number of colonies was calculated after $12 \mathrm{~h}$ of incubation at $37^{\circ} \mathrm{C}$.

\section{Cytokine assay}

Serum and peritoneal lavage fluid (PLF) samples were collected from 10 mice/group infected with $A$. baumannii and treated with FT, as described previously. The IL-6 assay range was $31.3-2000 \mathrm{pg} \mathrm{ml}^{-1}$ (determined by the lower limit of detection and the highest point of the standard curve). IL-6 levels in serum and PLF samples were analysed by ELISA (enzyme-linked immunosorbent assay) according to the manufacturer's instructions (Mouse IL-6 ELISA kit, Invitrogen by Thermo Fisher Scientific, Vienna, Austria). IL-6 levels in serum and PLF were determined in mice at 2, 4, and $14 \mathrm{~h}$ p.i. The levels of the cytokines IFN $\gamma$ (IFN gamma Mouse Uncoated ELISA Kit, Invitrogen by Thermo Fisher Scientific, Vienna, Austria) and TNF $\alpha$ (ELISA MAX $^{\mathrm{TM}}$ Deluxe Set Mouse TNF- $\alpha$, BioLegend, San Diego, USA) in serum and PLF were also assessed $2 \mathrm{~h}$ p.i. Optical densities were measured using a Multiskan EX plate reader at a wavelength of $450 \mathrm{~nm}$.

\section{Crystal violet biofilm assay}

The effect of FT on A. baumannii static biofilm formation was assayed in 96-well polystyrene plates. In brief, overnight cultures of $A$. baumannii were inoculated in LB broth and adjusted with shaking at $37^{\circ} \mathrm{C}$ to $0.5 \mathrm{McFarland}$ standards at $600 \mathrm{~nm}$ (OD600). Wells of a non-adherence, sterile 96-well plate were filled with $100 \mu \mathrm{l}$ of bacterial suspension with $250 \mu \mathrm{M}$ FT or without FT in triplicate. The effect of FT on biofilm formation was assessed at 2, 24, and $48 \mathrm{~h}$. During the 48-h incubation, FT was added twice, at the start of the experiment and $24 \mathrm{~h}$ later. Afterward, the content of each well was aspirated, rinsed three times with $200 \mu \mathrm{l}$ of sterile $\mathrm{diH}_{2} \mathrm{O}$, fixed with $96 \%$ ethanol and stained for 15 min with $0,1 \%$ crystal violet (CV) (Merck, Germany). For quantitative analysis, the dye bound to the adherent biofilm was resolubilized with $200 \mu \mathrm{l}$ of $96 \%$ ethanol. One hundred microlitres of solubilized $\mathrm{CV}$ was transferred to a new microtitre plate, and the optical density was measured by using a Multiskan EX plate reader at a wavelength of $540 \mathrm{~nm}\left(\mathrm{OD}_{540}\right)$.

Twenty-four-well plates were used for visual analysis. Biofilms were grown as described previously. Biofilm formation was assessed visually under a light microscope (Nikon Eclipse 50i (Nikon, Japan)) at a magnification of $\times 20$.

\section{Congo red biofilm assay}

Congo red (CR) can be used for direct measurement of biofilm matrix production or as an indirect measurement of intracellular c-di-GMP concentrations [9]. For this, the quantitative analysis of biofilm production was used as previously described. Briefly, the plates, after fixation with $96 \%$ ethanol, were stained for 15 min with $200 \mu$ of $0.1 \%$ CR (Merck, Germany). The plate was washed with water, emptied and left to dry. The dye bound to the adherent biofilm was resolubilized with $200 \mu \mathrm{l}$ of $96 \%$ ethanol. Quantitative analysis was carried out at a wavelength of $490 \mathrm{~nm}\left(\mathrm{OD}_{490}\right)$.

Twenty-four-well plates were used for visual analysis. Biofilms were grown and stained as described previously. A light microscope was used to visualize the production of polysaccharides in the biofilm matrix (Nikon Eclipse 50i (Nikon, Japan)) at a magnification of $\times 20$.

\section{Cell cytotoxicity assays}

HeLa cells (cultured in either 24- or 96-well plates) in RPMI-1640 medium supplemented with $10 \%$ fetal bovine serum (FBS) for $24 \mathrm{~h}$ were washed and covered with RPMI1640 containing $1 \%$ FBS. A. baumannii ABT-897-17 grown overnight in LB medium was subcultured in fresh LB and grown to the mid-log phase. HeLa cells were infected with mid-log-phase $A$. baumannii at an initial multiplicity of infection (MOI) of 1 . When measuring the release of lactate dehydrogenase $(\mathrm{LDH})$, plates were incubated for $24 \mathrm{~h}$ in the presence of FT or diluent in the controls and centrifuged at $1500 \mathrm{rpm}$ for $10 \mathrm{~min}$ to precipitate the bacteria. $\mathrm{LDH}$ release was measured using a commercial kit (CytoTox 96, Promega). The percentage of $\mathrm{LDH}$ release was calculated relative to the uninfected control, which was set as $0 \% \mathrm{LDH}$ release, and the cells lysed with Triton $\mathrm{X}$ 100 , which was set as $100 \%$ LDH release.

For staining with bromide ethidium, cells were cultured on coverslips in 24-well plates and infected as described above (MOI 1). Plates were incubated for 24 and $48 \mathrm{~h}$ in the presence of FT or diluent in the controls. Coverslips were washed with PBS, dried at room temperature, fixed with acetone for $30 \mathrm{~min}$ and stained for $10 \mathrm{~min}$ with bromide ethidium. Ethidium bromide penetration into cells and its binding to nuclei were visualized under a fluorescence microscope (Nikon Eclipse 50i) at a magnification of $\times 20$.

\section{Statistical analysis}

All experiments were performed using at least 4 samples per group and repeated 2 times. Significant differences in the data were determined using Mann-Whitney nonparametric 
two-tailed tests with GraphPad Prism Version 6. $P<0.05$ was considered statistically significant.

\section{Results}

\section{FT rescued animals in a murine model of $A$. baumannii-associated septicemia given in a prophylaxis regimen}

Previously, in preclinical studies, we showed that FT improved the outcome in severe antibiotic-resistant $P$. aeruginosa airway infection and systemic Salmonellas sp. infection when given in both prophylaxis and treatment regimens $[6,8]$. The effectiveness of prophylactic administration may be associated with the features of the pharmacokinetics of FT, which have been shown to maintain FT in the systemic circulation for extended periods and lead to effective tissue distribution in animals [7]. We also observed that after repeated per os administration to rats at a dose of $10 \mathrm{mg} \mathrm{kg}^{-1}$ for 6 days, the FT concentration in the blood increased from 1.3 to $26.57 \mathrm{ng} \mathrm{ml}^{-1}$ and in the lung, it increased from 11.37 to $41.52 \mathrm{ng} \mathrm{ml}^{-1}$ (data not shown).

In this study, we evaluated the antibacterial effect of FT given in the prophylaxis regimen in a murine model of $A$. baumannii-associated septicemia.

A. baumannii ABT-897-17 isolated from the blood of a patient with abdominal sepsis was used. DBA/2 mice were inoculated intraperitoneally with A. baumannii ABT-897-17 in two doses: $10^{8}$ and $5.0 \times 10^{7} \mathrm{CFU} /$ mouse $(n=4 \times 2$ replicates for each group). The mice were treated with 250 $\mathrm{mg} \mathrm{kg}^{-1}$ FT per os once per day for 4 days before infection. Survival rates were analysed within 5 days p.i. (Fig. 1)

As shown in Fig. 1, FT was associated with an increased survival of $50 \%$ of the mice after challenge with a dose of clinical isolate ABT-897-17 equal to $10^{8} \mathrm{CFU} / \mathrm{animal}$ (Fig. 1a) and $80 \%$ of infected mice treated with a dose of ABT-897-17 equal to $5 \times 10^{7} \mathrm{CFU} / \mathrm{animal}$ (Fig. 1b).
Mortality in the control group receiving the dose of $10^{8}$ CFU/animal was $100 \%$, and for those receiving the $5 \times 10$ CFU/animal dose, it was $60 \%$. These results showed that FT administered per os reduced the mortality of infected animals in the first 5 days p.i.

Next, we used a highly virulent clinical isolate of $A$. baumannii 52TS19. A. baumannii 52TS19 is at least $\sim 100$ fold more virulent than ABT-897-17 in DBA/2 mice when inoculated intraperitoneally [10]. For further experiments, we used a less sensitive strain of mice to study the effect of FT [11].

BALB/c mice were inoculated intraperitoneally with $A$. baumannii 52TS19 in two doses: $5.0 \times 10^{7}$ and $10^{7} \mathrm{CFU} /$ mouse ( $n=4 \times 2$ replicates for each group). The mice were treated with $250 \mathrm{mg} \mathrm{kg}^{-1}$ FT per os once per day for 4 days before infection. Survival rates were analysed within $24 \mathrm{~h}$ p.i. (Fig. 2).

As shown in Fig. 2, all the control mice succumbed to infection within $24 \mathrm{~h}$ after intraperitoneal inoculation with $5.0 \times 10^{7}$ or $10^{7} \mathrm{CFU} /$ animal. However, prophylactic treatment with FT ensured survival for $24 \mathrm{~h}$ in $50 \%$ of the mice after challenge with a dose of $5.0 \times 10^{7} \mathrm{CFU} /$ mouse clinical isolate 52TS19 (Fig. 2a) and $87.5 \%$ of the mice after challenge with a dose of $10^{7} \mathrm{CFU} /$ mouse (Fig. 2b). Observation of the mice continued for 5 days after infection. Animals that survived for the first $24 \mathrm{~h}$ survived up to 5 days. The results obtained showed that the prophylactic use of FT in septic infection caused by isolates of $A$. baumannii with different virulence led to increase survival of the mice.

\section{FT decreased bacterial loads in surviving murine models of $A$. baumannii-associated septicemia}

To characterize the effect of FT on bacterial load in mice, the number of bacteria in the lung and spleen homogenates and blood was counted. Animals infected with the A. baumannii ABT-897-17 clinical isolate at a dose of 5.0 $\times 10^{7}$ $\mathrm{CFU} /$ mouse were sacrificed on days 1 or 2 p.i. (Fig. 3).
Fig. 1 Prophylaxis treatment with FT improved the survival of DBA/2 mice infected with $A$. baumannii antibiotic-resistant clinical isolate ABT-897-17. a Mice infected with a dose of $10^{8}$ CFU/animal; b Mice infected with a dose of $5.0 \times 10^{7}$ CFU/animal. Mice were treated with $250 \mathrm{mg} \mathrm{kg}^{-1}$ of FT per os once a day for 4 days before infection. Black line-control, dotted line-FT-treated mice
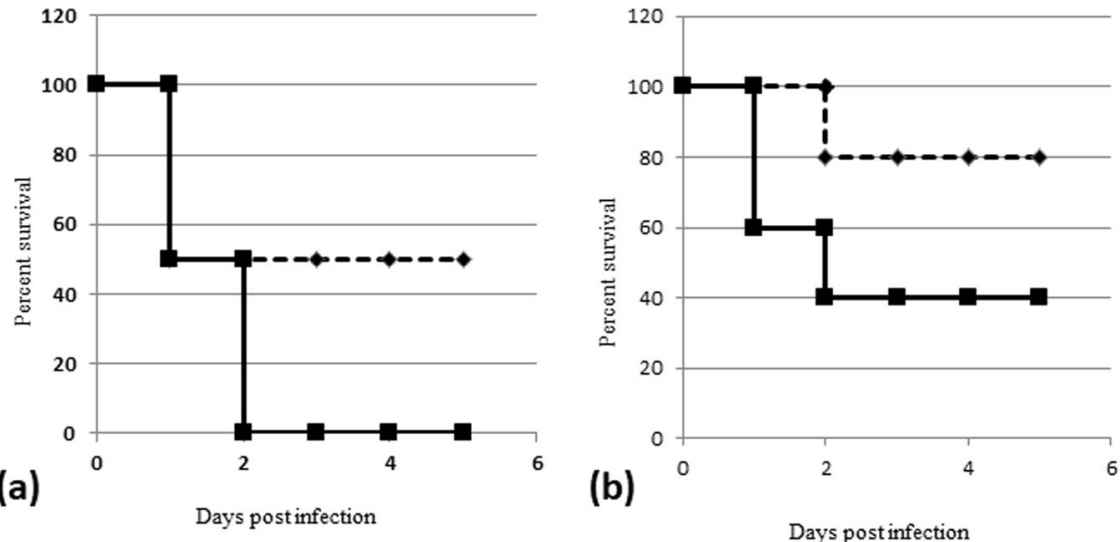
Fig. 2 Prophylaxis treatment Balb/c mice infected with $A$. baumannii antibiotic-resistant clinical isolate 52TS19. a Mice infected with a dose of $5.0 \times 10^{7}$ CFU/animal; b Mice infected with a dose of $10^{7} \mathrm{CFU} /$ animal. Mice were treated with $250 \mathrm{mg}$ $\mathrm{kg}^{-1}$ of FT per os once a day for 4 days before infection. Black line-control, dotted line-FTtreated mice with FT improves survival of
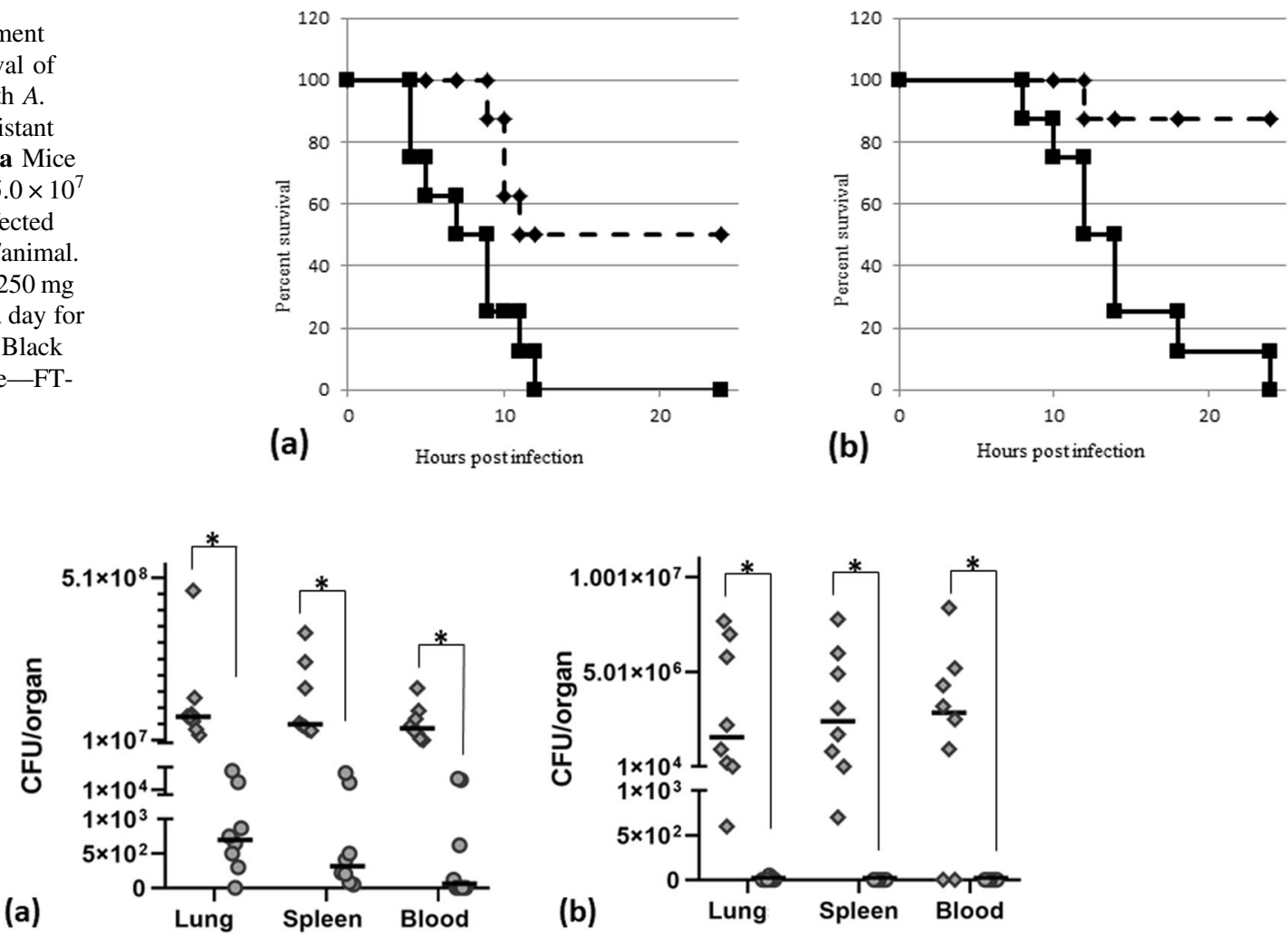

Fig. 3 The prophylaxis regimen with FT reduced A. baumanni loads after infection with antibiotic-resistant $A$. baumanni clinical isolate ABT-897-17. DBA/2 mice were infected with $5.0 \times 10^{7} \mathrm{CFU} /$ mouse of A. baumanni ABT-897-17. Mice were treated with $250 \mathrm{mg} \mathrm{kg}^{-1}$ of FT per os once a day for 4 days before infection. Bacterial loads in lungs,

As shown in Fig. 3, A. baumannii ABT-897-17 was found in all the organs of the control animals infected with a dose of $5.0 \times 10^{7} \mathrm{CFU} / \mathrm{animal}$ on the first day after infection. At this time point, administration of FT was shown to cause a decrease in bacterial burden in the lung, spleen, and blood by several orders of magnitude compared to the controls $(P \geq 0.05)$. Two days after infection, bacteria were also detected in all the organs of the control animals. Preventative treatment with FT resulted in almost complete elimination of A. baumannii ABT-897-17 in all the examined organs.

Next, we studied the effect of FT on reducing the bacterial load upon infection with the highly virulent $A$. baumannii 52TS19 strain. The study of lung and spleen homogenates and blood was carried out 2, 4, 14, and $20 \mathrm{~h}$ after intraperitoneal infection (Fig. 4).

FT prophylaxis treatment led to a decrease in the bacterial load in the blood after $2 \mathrm{~h}$ compared to the control group. However, after 4 and $14 \mathrm{~h}$, the number of bacteria in the analysed organs in the control and prophylactic groups did not differ significantly. By $20 \mathrm{~h}$ after infection, in the group of animals that had received FT prophylaxis, the

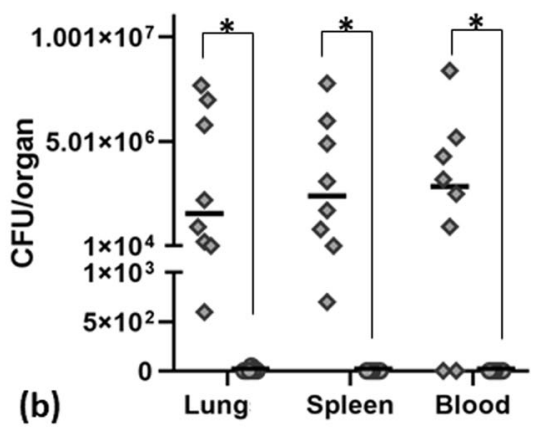

spleen, and blood of animals were analyzed on day 1 (a) and 2 (b) post-infection. Diamonds-mice not treated with FT, circles-mice treated with FT. The horizontal line represents the median CFU per group, each symbol represents one mouse. $* p<0.05$ relative to the control group at the corresponding time points

bacterial load in the studied organs was several orders of magnitude lower.

\section{FT affected the production of IL- 6 in peritoneal lavage fluid and serum}

We analysed the effect of prophylactic FT treatment on cytokine production by assessing cytokine levels in PLF and serum after $A$. baumannii intraperitoneal infection caused by injection of clinical isolate $52 \mathrm{TS} 19$ at $5 \times 10^{7}$ $\mathrm{CFU} /$ mouse. The mice were treated with $250 \mathrm{mg} \mathrm{kg}^{-1} \mathrm{FT}$ per os once per day for 4 days before infection. The mice were sacrificed 2, 4, or $14 \mathrm{~h}$ after bacterial infection $(n=10$ in each group, two separate experiments). The levels of key cytokines involved in the regulation of inflammation were determined in the PLF and serum.

Prophylaxis with FT resulted in a significant decrease in the concentration of IL- 6 in the peritoneal lavage of mice compared to the control at 2,4 , and $14 \mathrm{~h}$ after infection with A. baumannii $(P<0.05)$ (Fig. 5a). This finding indicated the limitation of the local inflammatory response, which was inhibited by FT. Determination of the level of IL-6 in the 
Fig. 4 The prophylaxis regimen with FT reduced A. baumanni loads after infection with antibiotic-resistant $A$. baumanni clinical isolate 52TS19. Balb/ c mice were infected with $10^{7}$ CFU/mouse of A. baumanni 52TS19. Mice were treated with $250 \mathrm{mg} \mathrm{kg}^{-1}$ of FT per os once a day for 4 days before infection. Bacterial loads in lungs, spleen, and blood of animals were analyzed at 2 (a), 4 (b), 14 (c), and 20 (d) hours. Diamondsmice not treated with FT, circles - mice treated with FT. The horizontal line represents the median CFU per group, each symbol represents one mouse. $* p<0.05$ relative to the control group at the corresponding time points
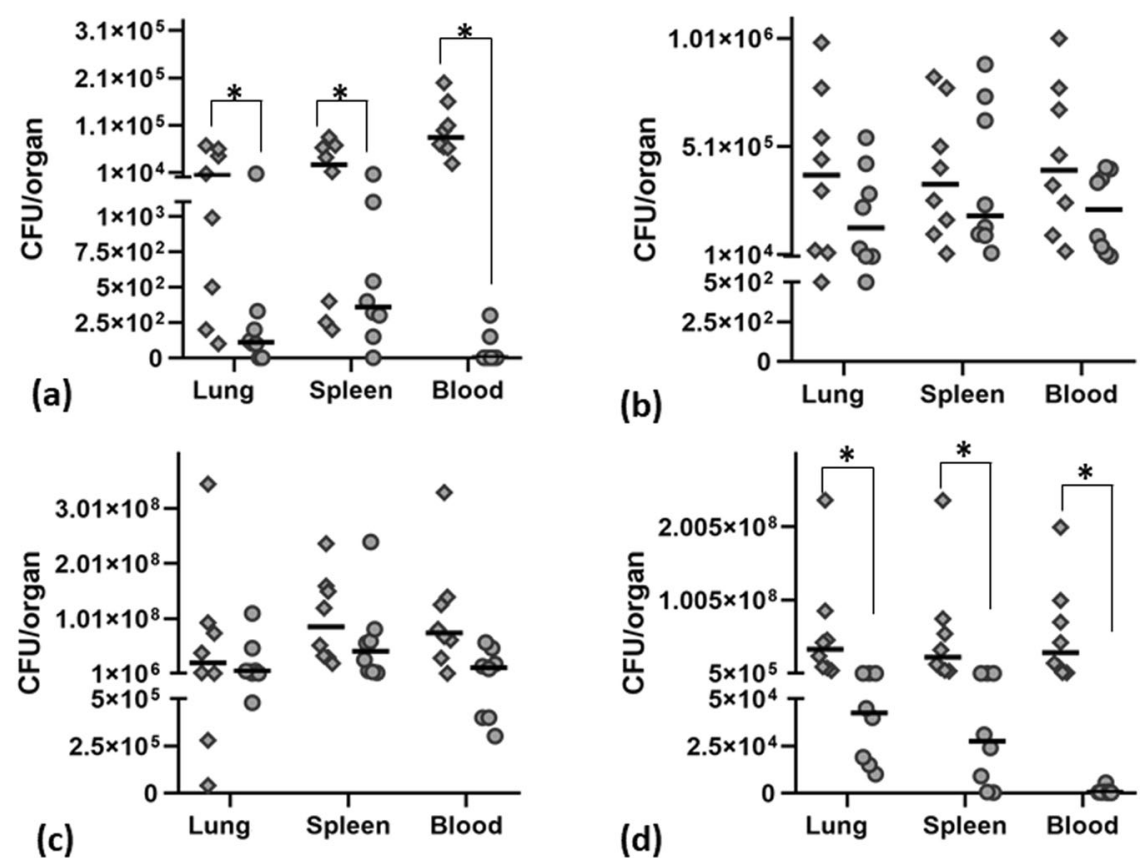

(a)
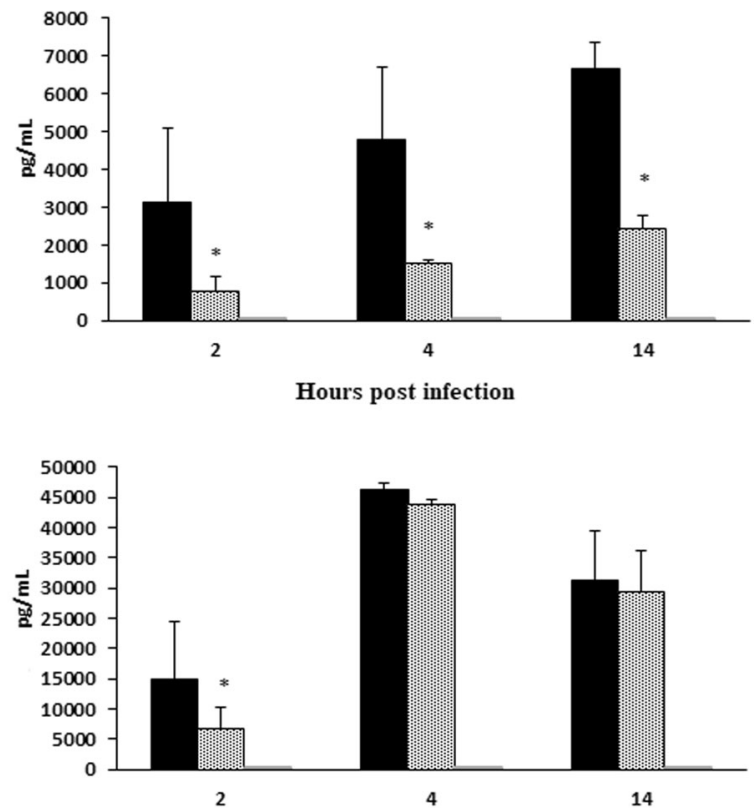

(b)

Hours post infection

Fig. 5 FT modulates the production of inflammatory cytokine IL-6 in peritoneal lavage fluid and serum. Balb/ $\mathrm{c}$ mice were infected with $10^{7} \mathrm{CFU} /$ mouse of A. baumanni 52TS19. Mice were treated with $250 \mathrm{mg} \mathrm{kg}^{-1}$ of FT per os once a day for 4 days before infection. IL-6 was tested in peritoneal lavage fluid (a) and serum (b) at 2, 4, and $14 \mathrm{~h}$ PI. Black bar, treatment with FT; dotted bar, untreated infected mice; gray bar, intact controls. $* p<0.05$ relative to the control group at the corresponding time points

serum showed a decrease in the group of mice treated with FT for only $2 \mathrm{~h}(P<0.05)$; no decrease in the systemic inflammatory response under the action of FT was observed in the other treatment periods (Fig. 5b). No alterations in TNF- $\alpha$ or IFN- $\gamma$ level were observed in the PLF or serum of any experimental groups compared to the control (data not shown).

\section{FT inhibited biofilm formation and maturation}

Furthermore, we decided to study the effect of FT on the formation of A. baumannii biofilms in vitro. In accordance with the previously shown mechanism of FT action on $P$. aeruginosa and Salmonella sp., FT in our study did not affect viability. We showed that FT at concentrations up to $500 \mu \mathrm{M}$ did not affect $A$. baumannii growth. The effect of FT on biofilm formation on the plastic surface was evaluated 2, 24, and $48 \mathrm{~h}$ after bacterial application. Biofilm development, as measured by biofilm biomass, was measured by optical absorbance $(540 \mathrm{~nm})$ using $\mathrm{CV}$, and CR staining was used to detect polysaccharide production in the biofilm matrix. The effect of FT on biofilm formation was assessed for two isolates of A. baumannii ABT-897-17 and 52 TS19.

FT had no significant effect on bacterial adhesion, since after $2 \mathrm{~h}$, the presence of FT showed no significant effect on planktonic cell attachment compared to the untreated control of both strains (Figs. S1, S2). However, at $24 \mathrm{~h}$, the inhibition of multilayer cell cluster accumulation under the action of FT was observed, and at $48 \mathrm{~h}$, the inhibitory effect against $A$. baumannii biofilm was more pronounced because the biofilm biomass did not increase in the presence of FT at a concentration of $250 \mu \mathrm{M}$ (Fig. S1a, b). FT at concentrations of 75 and $150 \mu \mathrm{M}$ did not inhibit biofilms at any time 
point. In these experiments, no difference was observed in bacterial growth in the presence or absence of FT (data not shown).

To assess the effect of FT on the production of the exopolysaccharide matrix, biofilms were stained with $\mathrm{CR}$, because $\mathrm{CR}$ can be utilized as a direct measurement of biofilm matrix production [9]. Measurement of the staining intensity of the biofilm matrix showed that FT reduced the production of matrix polysaccharides in a dose-dependent manner. (Fig. S2a, b). A significant decrease in the detectable matrix was observed after $48 \mathrm{~h}$ at an FT concentration of $75 \mu \mathrm{M}$.

Microscopic analysis of biofilm samples stained with CR to assess the level of production of the polysaccharide matrix showed a significant decrease in the density of the matrix layer in the biofilms after exposure to $250 \mu \mathrm{M}$ FT compared to the control samples after both 24 and $48 \mathrm{~h}$ for both isolates (Fig. S2b). The resulting images were correlated with the quantitation of biofilm polysaccharides obtained at a wavelength of $492 \mathrm{~nm}$.

\section{FT inhibited the $A$. baumannii-induced death of HeLa cells}

Next, we studied the effect of FT on A. baumannii ABT897-17 cytotoxicity in epithelial cells. After infecting HeLa cells with A. baumannii, we measured LDH levels and evaluated morphological changes in cells by ethidium bromide staining of DNA.

Measurements of LDH in control HeLa cells, cells incubated with $A$. baumannii for $24 \mathrm{~h}$, and infected cells treated with FT failed to reveal statistically significant differences; that is, A. baumannii did not damage the HeLa cell membrane under experimental conditions (data not shown).

Cells and nucleus were assessed after staining with ethidium bromide. Twenty-four hours after infection, $A$. baumannii did not destroy the monolayer of HeLa cells, but cells with nuclear condensation and fragmentation and cellular conglomerates were clearly identified. HeLa cells treated with FT at doses of 250 and $400 \mu \mathrm{M}$ maintained a typical, unchanged monolayer. After $48 \mathrm{~h}$, in cells infected with A. baumannii, ABT-897-17 changes were observed, such as cell shrinkage, membrane blebbing, rounding and detachment from the culture plate, and nuclear condensation and fragmentation. In addition, in the samples with the addition of 250 and $400 \mu \mathrm{M}$ FT, cells with a typical morphology were observed, but the cell monolayer was somewhat thinner compared to the control (Fig. S3). It was shown that A. baumannii caused morphological changes in cells that were characteristic of apoptosis. FT treatment at concentrations of 250 and $400 \mu \mathrm{M}$ significantly reduced cell death, which was more pronounced at $48 \mathrm{~h}$.

\section{Discussion}

In this study, we demonstrated that a small-molecule compound belonging to a class of 2,4-disubstituted-4H-[1,3,4]thiadiazine-5-ones, FT, when administered prophylactically suppressed acute intraperitoneal infection caused by virulent multidrug resistant $A$. baumannii clinical isolates.

The problem of ineffective treatment for nosocomial infections caused by multi-resistant bacteria, such as $A$. baumannii, $P$. aeruginosa, and $K$. pneumoniae, often leads to serious consequences, including the development of sepsis $[12,13]$. The complex therapy of patients infected with these bacteria includes different classes of antibiotics and anti-inflammatory drugs, but mortality, especially in ICUs, is very high $[14,15]$. Facing serious bacterial resistance, it is clear that new antibacterial strategies need to be rapidly developed and that relying on traditional antibiotics is no longer an effective practice [16]. We believe that a novel intervention strategy blocking the virulence of pathogens is the key to overcoming bacterial resistance [17]. These anti-virulence drugs could be effective as part of a complex therapy or used for prophylaxis.

In our work, we evaluated the prophylactic efficacy of the anti-virulence drug FT on the suppression of intraperitoneal infection caused by A. baumannii clinical isolates. When mice were challenged with two clinical isolates of $A$. baumannii, an acute infection developed, which caused $100 \%$ mortality within $24 \mathrm{~h}$ in the case of a highly virulent isolate or after 2 days in the case of a less virulent isolate. Prophylactic administration of FT to mice within 4 days prior to infection increased the post-challenge survival rate of the mice to 50 or $90 \%$, depending on the dose of inoculation and the virulence of the isolate.

The assessment of bacterial load in organs after FT prophylaxis showed a significant decrease in the number of Acinetobacter in the lung, blood, and spleen for a moderately virulent isolate on day 1 after infection and almost complete absence of the pathogen on day 2 . For highly virulent isolates under FT prophylaxis, a significant decrease in the number of bacteria in blood was observed $2 \mathrm{~h}$ post infection, which was not as pronounced after 4 or $14 \mathrm{~h}$. At $20 \mathrm{~h}$, the bacterial load in the organs of treated animals began to decrease, and bacteria were not detected in the blood at this time point. Most likely, the suppression of bacteraemia is crucial and determines the outcome of infection.

Rapid deterioration in the condition of infected animals and death during the first $12-20 \mathrm{~h}$ after infection with a virulent strain indicates the development of septic shock. We assessed the level of the key proinflammatory cytokine IL-6 in serum and peritoneal lavage. At all analysed time points, that is, 2, 4, and $14 \mathrm{~h}$ post infection, local IL-6 levels were decreased in the FT-treated mice, indicating a 
prophylactic effect induced by reducing pathological local inflammation. In addition, the assessment of the effect of FT on systemic inflammation and the detection of IL-6 in the serum of mice showed a significant decrease only $2 \mathrm{~h}$ after infection. It can be assumed that all the most significant processes of the inflammatory response, which were affected by FT, are characteristic of the earliest stage of the interaction of the pathogen with the immune system. Future studies will aim to address this presumption.

To further characterize the effect of FT on A. baumannii virulence, we assessed its effect on biofilm formation in vitro. FT suppressed the formation of biofilm biogenesis in a dose-dependent manner, and to a greater extent, this suppression was associated with the stage of mature biofilm formation during the formation of the polysaccharide matrix. This observation confirms our previously obtained data on the suppression of $P$. aeruginosa biofilms formed on the MDCK cell surface in the presence of FT.

The ability of A. baumannii to form biofilms determines its extreme resistance to antibiotics and many other antimicrobial agents as well as its ability to evade host defences $[18,19]$. Several virulence factors are involved in biofilm formation of $A$. baumannii, such as the outer membrane protein A (OmpA), biofilm-associated protein, chaperoneusher pilus, extracellular exopolysaccharide, a twocomponent system (BfmS/BfmR), poly- $\beta-(1,6)-N$-acetyl glucosamine and a quorum sensing system [20-22]. Among these factors, OmpA of A. baumannii plays a dominant role in forming stable biofilms on plastic surfaces.

We have not identified the mechanism of suppression of A. baumannii biofilm by FT; nevertheless, without affecting the viability of Acinetobacter, after $48 \mathrm{~h}$, FT treatment significantly reduced the biomass of the biofilm that had formed on the surface of the plastic, as well as the amount of the polysaccharide matrix.

In in vitro experiments, we also observed the effect of FT on HeLa cell death caused by A. baumannii. The cytotoxicity of A. baumannii appears to be unrelated to T3SS, given that in silico analysis did not reveal any T3SS components in this bacterium. Recently, it was shown that, similar to many other Gram-negative bacteria, A. baumannii produces outer membrane vesicles (OMVs) as toxic protein delivery platforms [23, 24]. One of the most abundant proteins associated with OMVs is OmpA, which has been extensively studied for its role in A. baumannii virulence. It has been shown that the OmpA protein is a multifunctional virulence factor participating in A. baumannii biofilm formation, adhesion to epithelial cells, cytotoxicity, inhibition of host immune response and resistance to various antimicrobial drugs [25-28]. A. baumannii packages OmpA into OMVs, and the OMV-mediated delivery of OmpA to host cells induces host cell death via both mitochondrial and nuclear targeting [29, 30].
In our experiments, we evaluated the death of HeLa cells caused by A. baumannii clinical isolates. We observed morphological signs of cell death characteristic of apoptosis, including cell rounding, fragmentation of nuclei, detachment of cells, but we did not observe disruption to the integrity of the cell membrane [31, 32]. FT effectively suppressed cell death within $48 \mathrm{~h}$ of bacterial contact with HeLa cells.

We continued to study the mechanism of FT action on Acinetobacter infection. For FT, a specific effect on the type three secretion system has been previously shown in Pseudomonas, Salmonella, and Chlamydia [4, 6-8, 33]. It is likely that FT has another target in A. baumannii because they have not yet been shown to have the T3SS. FT is clearly an inhibitor of a significant virulence factor that is key for septic infection, as well as an inhibitor of biofilm formation and bacterial interaction with cells. In addition, in accordance with the mechanism of action previously shown for different pathogens, FT does not affect the viability of bacteria.

Thus, our results clearly show a prophylactic effect of the small-molecule compound in a mouse model of septicemia induced by clinically antibiotic-resistant $A$. baumannii isolates. The data obtained to date indicate that FT suppresses the virulence of a number of gram-negative bacteria, including pathogens with different patterns of interaction with the host cell. Further study of FT will make it possible to develop a broad-spectrum anti-virulence drug.

A formulated drug product with FT as an active ingredient, named Ftortiazinon, is undergoing research in phase 2 clinical trials in Russia. This study was planned to evaluate the safety and efficacy of the drug Ftortiazinon at the doses of 300,600 , and $1200 \mathrm{mg}$ per day in combination with the drug Cefepime in comparison with placebo in combination with the drug Cefepime in the treatment of hospitalized adult patients with complicated urinary tract infections caused by $P$. aeruginosa. (NCT03638830). Preliminary results show the efficacy of 600 and $1200 \mathrm{mg} / \mathrm{day}$ of FT in the clinical cure and microbiological elimination of $P$. aeruginosa and E. coli. Higher doses are required for the prevention and treatment of $A$. baumannii infection. In this regard, a phase 1 clinical trial was conducted to evaluate the safety of FT at the doses of 1800 and $2400 \mathrm{mg}$ /day. In this regard, a phase 1 clinical trial was conducted to evaluate the safety of the doses of FT 1800 and $2400 \mathrm{mg}$ /day which showed a good safety profile and no serious adverse effects.

\section{Compliance with ethical standards}

Conflict of interest The authors declare no competing interests.

Publisher's note Springer Nature remains neutral with regard to jurisdictional claims in published maps and institutional affiliations. 


\section{References}

1. Lima WG, Brito JCM, da Cruz Nizer WS. Ventilator-associated pneumonia (VAP) caused by carbapenem-resistant Acinetobacter baumannii in patients with COVID-19: two problems, one solution? Med Hypotheses. 2020;144:110139.

2. Abdi SN, Ghotaslou R, Ganbarov K, Mobed A, Tanomand A, Yousefi $\mathrm{M}$, et al. Acinetobacter baumannii efflux pumps and antibiotic resistance. Infect Drug Resistance. 2020;13:423-34.

3. Vazquez Guillamet C, Kollef MH. Acinetobacter pneumonia: improving outcomes with early identification and appropriate therapy. Clin Infect Dis. 2018;67:1455-62.

4. Koroleva EA, Kobets NV, Zayakin ES, Luyksaar SI, Shabalina LA, Zigangirova NA. Small molecule inhibitor of type three secretion suppresses acute and chronic Chlamydia trachomatis infection in a novel urogenital Chlamydia model. Biomed Res Int. 2015;2015:484853.

5. Zigangirova NA, Zayakin ES, Kapotina LN, Kost EA, Didenko LV, Davydova DY, et al. Development of Chlamydial Type III Secretion System Inhibitors for Suppression of Acute and Chronic Forms of Chlamydial Infection. Acta Nat. 2012;4:87-97.

6. Sheremet AB, Zigangirova NA, Zayakin ES, Luyksaar SI, Kapotina LN, Nesterenko LN, et al. Small Molecule Inhibitor of Type Three Secretion System Belonging to a Class 2,4-disubstituted-4H-[1,3,4]-thiadiazine-5-ones Improves Survival and Decreases Bacterial Loads in an Airway Pseudomonas aeruginosa Infection in Mice. Biomed Res Int. 2018;2018:5810767.

7. Nesterenko LN, Zigangirova NA, Zayakin ES, Luyksaar SI, Kobets $\mathrm{NV}$, Balunets DV, et al. A small-molecule compound belonging to a class of 2,4-disubstituted 1,3,4-thiadiazine-5-ones suppresses Salmonella infection in vivo. J Antibiot. 2016;69:422-7.

8. Zigangirova NA, Nesterenko LN, Sheremet AB, Soloveva AV, Luyksaar SI, Zayakin ES, et al. Fluorothiazinon, a smallmolecular inhibitor of T3SS, suppresses Salmonella oral infection in mice. J Antibiotics. 2021;74:244-54.

9. Jones CJ, Wozniak DJ. Congo red stain identifies matrix overproduction and is an indirect measurement for c-di-GMP in many species of bacteria. In: c-di-GMP Signaling. New York, NY: Humana Press; 2017. p. 147-156.

10. Samet SJ, Tompkins SM. Influenza pathogenesis in genetically defined resistant and susceptible murine strains. Yale J Biol Med. 2017;90:471-9. Sep 25PMID: 28955185; PMCID: PMC5612189

11. Harris G, KuoLee R, Xu HH, Chen W. Acute intraperitoneal infection with a hypervirulent Acinetobacter baumannii isolate in mice. Sci Rep. 2019;9:1-12.

12. Motbainor H, Bereded F, Mulu W. Multi-drug resistance of blood stream, urinary tract and surgical site nosocomial infections of Acinetobacter baumannii and Pseudomonas aeruginosa among patients hospitalized at Felegehiwot referral hospital, Northwest Ethiopia: a cross-sectional study. BMC Infect Dis. 2020;20:92.

13. Rivera-Espinar F, Machuca I, Tejero R, Rodríguez J, Mula A, Marfil E, et al. Impact of KPC Production and high-level meropenem resistance on all-cause mortality of ventilator-associated pneumonia in association with Klebsiella pneumoniae. Antimicrobial Agents Chemother. 2020;64:e02164-19.

14. Pogue JM, Kaye KS, Veve MP, Patel TS, Gerlach AT, Davis SL, et al. Ceftolozane/tazobactam vs polymyxin or aminoglycosidebased regimens for the treatment of drug-resistant Pseudomonas aeruginosa. Clin Infect Dis. 2020;71:304-10.

15. Khawcharoenporn T, Chuncharunee A, Maluangnon C, Taweesakulvashra T, Tiamsak P. Active monotherapy and combination therapy for extensively drug-resistant Pseudomonas aeruginosa pneumonia. Int J Antimicrobial Agents. 2018;52:828-34.
16. Rezzoagli C, Archetti M, Mignot I, Baumgartner M, Kummerli R, Schulenburg H. Combining antibiotics with antivirulence compounds can have synergistic effects and reverse selection for antibiotic resistance in Pseudomonas aeruginosa. PLoS Biol. 2020;18:e3000805.

17. Buroni S, Chiarelli LR. Antivirulence compounds: a future direction to overcome antibiotic resistance? Future Microbiol. 2020;15:299-301.

18. Penesyan A, Nagy SS, Kjelleberg S, Gillings MR, Paulsen IT. Rapid microevolution of biofilm cells in response to antibiotics. NPJ Biofilms Microbiomes. 2019;5:1-14.

19. Gordon NC, Wareham DW. Multidrug-resistant Acinetobacter baumannii: mechanisms of virulence and resistance. Int J Antimicrobial Agents. 2010;35:219-26.

20. Ghasemi E. Phenotypic and genotypic investigation of biofilm formation in clinical and environmental isolates of Acinetobacter baumannii. Arch Clin Infect Dis. 2018;13:4.

21. Thummeepak R, Kongthai $P$, Leungtongkam U, Sitthisak S. Distribution of virulence genes involved in biofilm formation in multi-drug resistant Acinetobacter baumannii clinical isolates. Int Microbiol. 2016;19:121-9.

22. Zeighami H, Valadkhani F, Shapouri R, Samadi E, Haghi F. Virulence characteristics of multidrug resistant biofilm forming Acinetobacter baumannii isolated from intensive care unit patients. BMC Infect Dis. 2019;19:1-9.

23. Jin JS, Kwon SO, Moon DC, Gurung M, Lee JH, Kim SI, et al. Acinetobacter baumannii secretes cytotoxic outer membrane protein A via outer membrane vesicles. PloS One. 2011;6:e17027.

24. Jha C, Ghosh S, Gautam V, Malhotra P, Ray P. In vitro study of virulence potential of Acinetobacter baumannii outer membrane vesicles. Microb Pathogenesis. 2017;111:218-24.

25. Lee H-W, Koh YM, Kim J, Lee JC, Lee YC, Seol SY, et al. Capacity of multidrug-resistant clinical isolates of Acinetobacter baumannii to form biofilm and adhere to epithelial cell surfaces. Clin Microbiol Infect. 2008;14:49-54.

26. Gaddy JA, Tomaras AP, Actis LA. The Acinetobacter baumannii 19606 OmpA protein plays a role in biofilm formation on abiotic surfaces and in the interaction of this pathogen with eukaryotic cells. Infect Immun. 2009;77:3150-60.

27. Lee JC, Koerten H, van den Broek P, Beekhuizen H, Wolterbeek $\mathrm{R}$, van den Barselaar M, et al. Adherence of Acinetobacter baumannii strains to human bronchial epithelial cells. Res Microbiol. 2006;157:360-6.

28. Jun SH, Lee JH, Kim BR, Kim SI, Park TI, Lee JC, et al. Acinetobacter baumannii outer membrane vesicles elicit a potent innate immune response via membrane proteins. PloS One. 2013;8:e71751.

29. Lee JS, Choi CH, Kim JW, Lee JC. Acinetobacter baumannii outer membrane protein A induces dendritic cell death through mitochondrial targeting. J Microbiol. 2010;48:387-92.

30. An Z, Huang X, Zheng C, Ding W. Acinetobacter baumannii outer membrane protein A induces HeLa cell autophagy via MAPK/JNK signaling pathway. Int J Med Microbiol. 2019;309:97-107.

31. Cano V, Moranta D, Llobet-Brossa E, Bengoechea JA, Garmendia J. Klebsiella pneumoniae triggers a cytotoxic effect on airway epithelial cells. BMC Microbiol. 2009;9:1-9.

32. Jayathilaka E, Rajapaksha DC, Nikapitiya C, De Zoysa M, Whang I. Antimicrobial and anti-biofilm peptide octominin for controlling multidrug-resistant Acinetobacter baumannii. Int $\mathrm{J}$ Mol Sci. 2021;22:5353.

33. Zigangirova NA, Kost EA, Didenko LV, Kapotina LN, Zayakin ES, Luyksaar SI, et al. A small-molecule compound belonging to a class of 2,4-disubstituted 1,3,4-thiadiazine-5-ones inhibits intracellular growth and persistence of Chlamydia trachomatis. J Med Microbiol. 2016;65:91-8. 